\title{
Modelo para predição de ações e inferência de situações de risco em ambientes sensíveis ao contexto
}

\author{
Alfredo Del Fabro Neto ${ }^{1}$, Bruno Romero de Azevedo ${ }^{1}$, Rafael Bouefleuer ${ }^{1}$, \\ João Carlos D. Lima ${ }^{2}$, Iara Augustin ${ }^{1}$ \\ ${ }^{1}$ Programa de Pós-Graduação em Informática \\ ${ }^{2}$ Departamento de Linguagens e Sistemas Computacionais \\ Universidade Federal de Santa Maria (UFSM) \\ \{alfredodfn, rafaboufler\}@redes.ufsm.br \\ \{brunodea, caio, august\}@inf.ufsm.br
}

\begin{abstract}
Human activities of daily living may have an associated harm risk for the one who is performing it. This paper proposes an approach for action prediction and detection of risk situations based on the physiological context changes caused by them by using the Hyperspace Analogous to Context. Testes were made for the action prediction, with a precision of 78,69\%, and for the detection of risk situations, with a precision of 98,94\%.
\end{abstract}

Resumo. A atividade cotidiana humana pode ter um risco de dano associado para quem a está realizando. Este trabalho propõe uma abordagem para a predição de ações e detecção de situações de risco, baseando-se nas mudanças de contexto fisiológico provocadas por elas através do modelo Hiperespaço Análogo ao Contexto. Testes foram realizados para a predição de ações, $e$ obtiveram precisão de 78,69\%, e para a detecção de situações de risco, precisão de $98,94 \%$.

\section{Introdução}

O aumento da disponibilidade de sensores de baixo custo e de tamanho reduzido, bem como a consolidação de tecnologias de dispositivos móveis, tem possibilitado o avanço de pesquisas na área de computação ubíqua e pervasiva [Silva et al. 2012]. Atualmente, os dispositivos móveis são equipados com sensores que permitem capturar diferentes dados capazes de representar o usuário e o ambiente em que ele está inserido. Avanços da microeletrônica contribuíram para pesquisas na criação de ambientes inteligentes tais como casas inteligentes, sistemas de healthcare e sistemas de recomendação de serviços. Assim, para que se possa fornecer serviços personalizados como os citados, é necessário compreender o modo com o qual o usuário interage com o ambiente. Para tal, os sistemas devem ter acesso aos dados relacionados ao contexto em que os usuários estão inseridos. E, em especial, analisar a interação entre um usuário e o ambiente através da detecção das atividades realizadas pelo indivíduo [Mocanu and Florea 2011].

Portanto, a detecção de atividades é um ponto crucial em ambientes inteligentes, visto que é parte constituinte do contexto no qual o usuário está inserido [Lu and Fu 2009, Crispim-Junior et al. 2012]. Neste sentido, a detecção de situações de 
risco provenientes de uma atividade apresenta papel igualmente importante, uma vez que, além de permitir a obtenção de informação contextual sobre a situação do usuário enquanto executa uma atividade, ela permite determinar a qualidade da interação entre o usuário e o ambiente. Além disso, a detecção de situações de emergência (ou de risco) possibilita que a própria informação contextual seja aperfeiçoada de forma a estabelecer um ciclo, onde cada atividade realizada pelo usuário aprimora o entendimento do contexto pessoal e a relação do mesmo com o ambiente [Mocanu and Florea 2011].

Este trabalho apresenta um modelo para determinar situações de risco baseado nas ações realizadas pelo usuário. Através da análise do comportamento normal do usuário e com base na Teoria da Atividade (TA) [Vygotsky 1980] e no modelo contextual Hiperespaço Análogo ao Contexto (HAC) [Rasch 2013], o modelo proposto é capaz de inferir a probabilidade de execução das próximas ações a serem realizadas. Já a determinação de situações de risco é realizada através do monitoramento de dados fisiológicos enquanto o usuário realiza a atividade. Desta forma, é possível associar uma situação (de risco) a cada ação ou atividade a ser desenvolvida com base no comportamento usual do usuário. Este trabalho se insere no âmbito do projeto para detecção de situações de risco Activity Project, o qual tem por base um middleware sensível ao contexto e o modelo proposto aqui está incorporado à camada Gerenciador de Atividades desse middleware [Del Fabro Neto et al. 2013].

Este artigo está organizado da seguinte forma: na seção 2 é apresentada a fundamentação teórica necessária para o entendimento do modelo proposto; na seção 3 são apresentados trabalhos relacionados à predição de ações e detecção de riscos; na seção 4 é apresentado o modelo proposto e seu funcionamento; na seção 5 são realizadas as avaliações da predição de ações e da detecção de situação de risco; e na seção 6 são apresentados as considerações finais e os trabalhos futuros.

\section{Fundamentação Teórica}

\subsection{Teoria da Atividade (TA)}

Com base na TA, uma atividade é composta por ações que são consideradas unidades atômicas, ou seja, não podem ser divididas em mais ações e sua noção básica é a de que o sujeito está participando de uma atividade porque quer atingir algum objetivo específico [Vygotsky 1980]. Seu interesse está centrado sobre o objeto de uma atividade que ele deseja utilizar e/ou modificar a fim de alcançar um resultado esperado. A interação entre o sujeito e o objeto é mediado por artefatos. Assim, um triângulo básico entre sujeito (subject), objeto (object) e mediação por artefatos (artifact) se forma (Figura 1).

Embora seja possível o reconhecimento de atividades baseado no modelo básico da $\mathrm{T} \Lambda$, ele não contempla todos os componentes que influenciam esse reconhecimento. $\mathrm{O}$ sujeito também possui contextos sociais e culturais que devem ser levados em consideração na mediação com a comunidade em que ele está inserido. Por esse motivo, a Teoria da Atividade Histórico Cultural (TAHC), apresentada na Figura 1, abrange o componente comunidade (community) mediada por regras (rules) e divisão do trabalho (division of labor) [Kuutti 1996]. Devido a dificuldade de representar esses conceitos computacionalmente, a taxonomia de contexto (Figura 2) foi desenvolvida de forma a proporcionar uma visão pragmática da construção dos artefatos e incorpora, aos sistemas sensíveis ao contexto, os conceitos gerais da Teoria da Atividade [Mikalsen and Kofod-Petersen 2004]. 


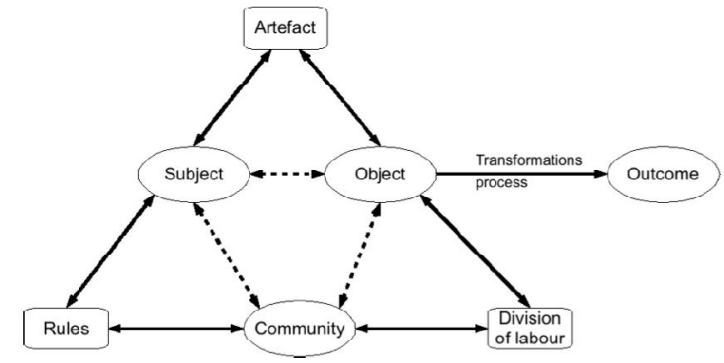

Figura 1. Teoria da Atividade Histórico-Cultural (TAHC) [Kuutti 1996].

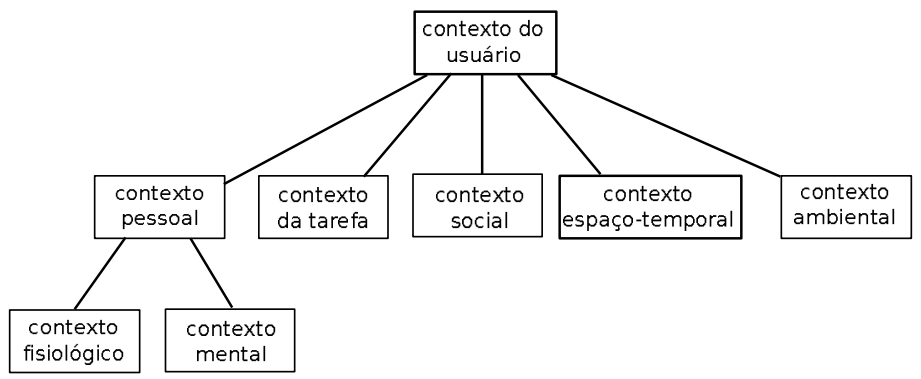

Figura 2. Taxonomia de Contexto. Fonte: [Mikalsen and Kofod-Petersen 2004]

\subsubsection{Composição das atividades}

Este trabalho utiliza a TA para modelar contextualmente uma atividade. Entretanto, como nessa, o relacionamento entre ações e como elas compõe uma atividade não são definidos do ponto de vista computacional, é necessário a definição da estrutura pela qual uma atividade é composta para uma modelagem do ponto de vista sistêmico. Para isso, Naeem et al. (2007) propuseram um modelo hierárquico de atividades para atividades da vida cotidiana (ADL) que pode ser estendido para qualquer sistema que defina uma atividade como uma composição de ações. Neste modelo, as ADLs são compostas de diversas ações e uma ADL pode compor outra ADL de nível superior. Para tanto, elas são elaboradas em uma linguagem de representação de planos hierárquica, denominada $\Lambda$ sbru, específica de tarefas e orientada à intenção. Em Asbru, uma ADL pode ser classificada como obrigatória ou opcional. Se uma ADL tem sub-objetivos (ou sub-atividades, ou ações) que são classificadas como obrigatórias, estas ações devem ser executadas antes da ADL ser rotulada como executada. Se for opcional, a ação não precisa ser necessáriamente executada para a atividade ser reconhecida.

Além disso, ações podem ser ordenadas de diferentes maneiras; por exemplo, de forma sequencial (em ordem rigorosa de acontecimento), paralela (executadas simultaneamente), em qualquer ordem (mas apenas uma ação pode ser executada em um momento) e não ordenada (executado sem sincronização). A partir dessas classificações, é possível desenvolver um modelo para cada uma das atividades que o sistema detecta e, dessa forma, definir se a atividade tem restrições de ordem de execução de ações ou de obrigatoriedade. Por último, é importante ressaltar que o modelo permite acrescentar restrições temporais, permitindo implementar uma janela de tempo para a detecção da atividade. Essa restrição temporal pode ser aplicada à ADL ou a cada ação que a compõe. 


\subsection{Hiperespaço Análogo ao Contexto (HAC)}

Para detectar atividades humanas é necessário detectar os atributos de contexto que ocorrem em um ambiente pervasivo. Para este propósito, optou-se por utilizar o modelo HAC, o qual utiliza múltiplas dimensões para caracterizar o contexto com valores que podem variar entre limiares pré-definidos. Assim, se um valor estiver fora do limiar para um atributo de contexto, uma ação pode ser tomada. O HAC apresenta uma sintaxe bem definida e define operações que permitem lidar com os dados de contexto.

Contudo, sua principal vantagem é a possibilidade de capturar todas as mudanças de contexto, permitindo o entendimento do comportamento do usuário. Dessa forma, as informações históricas de contexto do usuário e do ambiente podem ser usadas para determinar o risco de cada ação que compõe a atividade em desenvolvimento, bem como o risco nas possíveis ações futuras inferidas. Assim, se um usuário realizar uma ação que provoque uma mudança de contexto específica, pode-se avaliar se tal mudança acarretaria em uma situação de risco. Realizar a simulação de mudanças de contexto permite que cenários que ainda não aconteceram sejam verificados com o intuito de prever, probabilisticamente, se uma determinada ação ocasionará uma situação de risco a partir do contexto gerado. Este trabalho utiliza as mudanças de contexto para inferir se a alteração de determinado atributo de contexto pode resultar em uma mudança de contexto futura prejudicial ao usuário, ou seja, se ela o coloca em uma situação de risco.

\section{Sistemas para predição e inferência de situações de risco}

Curone et al. (2010) propuseram um sistema de classificação de situações de emergência para pessoas que arriscam suas vidas no cumprimento do dever, como bombeiros e socorristas da Defesa Civil. Os operadores são equipados com dois sensores nas roupas de proteção, um acelerômetro e um sensor ECG (eletrocardiograma). O sistema é composto por um classificador capaz de reconhecer diversos estados do usuário que correspondem a diversas atividades cotidianas em tempo real. Testes foram conduzidos em laboratório, e o sistema apresentado obteve cerca de $88,8 \%$ de precisão na classificação das atividades.

Wang et al. (2014) propõe uma abordagem distribuída que emprega os recursos de computação e armazenamento de cada nó da rede de sensores sem fio para detectar atividades anormais. No trabalho, uma atividade é definida como a combinação de trajetória e duração, e uma atividade anormal é definida como a atividade que desvia significativamente dos trajetos e durações das atividades normais. Para determinar o comportamento normal do indivíduo, os autores realizaram a mineração de padrões frequentes para encontrar os padrões de atividades normais, considerando sua duração e trajetória. Assim, se a frequência de um conjunto de itens (neste caso, considera-se como uma atividade) exceder o limite mínimo definido, trata-se de uma atividade normal. Caso contrário, a atividade é considerada anormal. Em um ambiente simulado por um software de autoria de Wang et al. (2014), a precisão foi de 96,2\%. Para testes em ambiente real, o valor de precisão não foi fornecido pelos autores.

Gil-Quijano e Sabouret (2010) apresentam uma proposta para predição de atividades domésticas em uma casa inteligente. O objetivo dos autores é adaptar o comportamento das aplicações da casa a partir das atividades humanas previstas, a fim de corrigir o comportamento de aparelhos e preparar os cômodos para receber as pessoas em condições mais agradáveis a elas. A proposta de predição de atividades baseia-se na construção de 
um grafo direcional para cada ocupante a partir da análise estatística das atividades executadas por ele. Os nós do grafo representam as tarefas e as arestas representam a sequência de execução entre duas tarefas, sendo que elas possuem atreladas a si a probabilidade de execução de sua sequência. Assim, uma vez que cada tarefa é realizada em um dado ambiente, é possível prever o próximo deslocamento no grafo a partir da tarefa atual. Caracterizando, assim, a predição de atividades.

\section{Modelo proposto}

O modelo proposto neste trabalho representa a camada Gerenciador de Atividades do Activitiy Project e objetiva realizar diferentes tarefas, sendo elas: $(i)$ detectar ações e atividades; (ii) associar uma situação de risco a cada uma delas; (iii) e inferir as ações e atividades futuras, para que seja possível a predição de situações de risco. A estrutura do modelo apresentada na Figura 3 funciona da seguinte forma: após receber os dados agregados de sensores, o primeiro passo é reconhecer a ação que está sendo executada e, a partir desta ação e com base no histórico de ações e atividades realizados pelo usuário, inferir a próxima ação a ser executada.

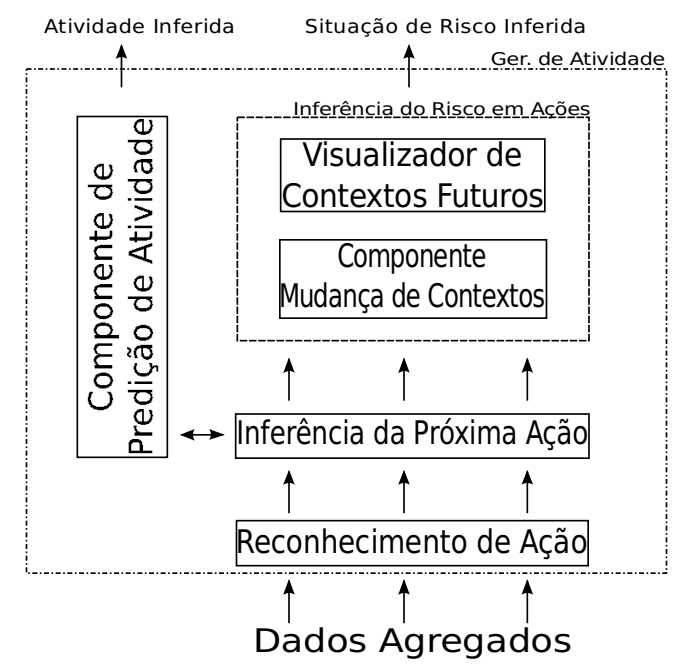

Figura 3. Modelo proposto para a camada Gerenciador de Atividades.

De posse da próxima ação a ser detectada, dois processos distintos são iniciados: $(i)$ o Componente de Predição de Atividade recebe a provável próxima ação e assume que ela de fato ocorreu, e solicita ao componente responsável pela Inferência da Próxima Ação uma nova ação futura, utilizando como ação atual a que foi detectada anteriormente. Dessa forma, o Componente de Predição de Atividade repete esse processo até que uma sequência de ações que representa uma atividade seja reconhecida (importante para o Activity Project devido à camada Classificador SRK abordada no artigo [Del Fabro Neto et al. 2014]). Para clarificar este processo, pode-se imaginar que o componente Reconhecimento de Ação reconheceu a ação atual $a_{1}$, e o componente Inferência da Próxima Ação detectou que a provável próxima ação a ser executada pelo usuário é a ação $a_{2}$. O que o Componente de Predição de Atividade faz é solicitar ao componente Inferência da Próxima Ação que ele calcule a próxima ação provável tendo como ação atual base a ação $a_{2}$. O processo utilizado para realizar essa inferência é explicado na seção 4.2; (ii) além disso, o sub-sistema Inferência do Risco em Ações associa 
uma situação de risco a cada ação inferida, com base nas mudanças de contexto e nos contextos futuros gerados por essas mudanças. Cada um desses componentes é explicado nas próximas subseções.

\subsection{Reconhecimento de Ação}

O componente Reconhecimento de Ação recebe os dados agregados de cada tipo de sensor e, com base em algoritmos de classificação, compara os dados recebidos com os dados já classificados para cada ação cadastrada no sistema. Em suma, o conjunto de dados de sensores será classificado de acordo com a similaridade que possui com os dados cadastrados para cada uma das ações. Dernbach et al. (2012) elencou seis algoritmos de aprendizagem de máquina para classificação baseada em dados brutos de sensores para o reconhecimento de ações: Multilayer Perceptron, Naive Bayes, Bayesian network, Decision Table, BestFirst Tree e Kstar. Os autores utilizaram o software WEKA para aplicação dos algoritmos sobre os dados brutos, com parâmetros padrões associados a cada um dos classificadores e aplicados aos dados após sofrerem um processo de extração de características. O algoritmo que conseguiu classificar o maior número de amostras corretamente foi o Kstar e, portanto, foi o algoritmo adotado para o modelo.

\subsection{Inferência da próxima ação do usuário}

A inferência da próxima ação a ser desempenhada pelo usuário é baseada no histórico de suas ações já executados por refletir o seu comportamento. Para tanto, o algoritmo 1 baseia-se na busca por padrões de atividades no histórico $H$ do usuário, ou seja, a busca de determinadas sequências de atividades com o intuito de descobrir qual a próxima ação que provavelmente será executada após esse padrão.

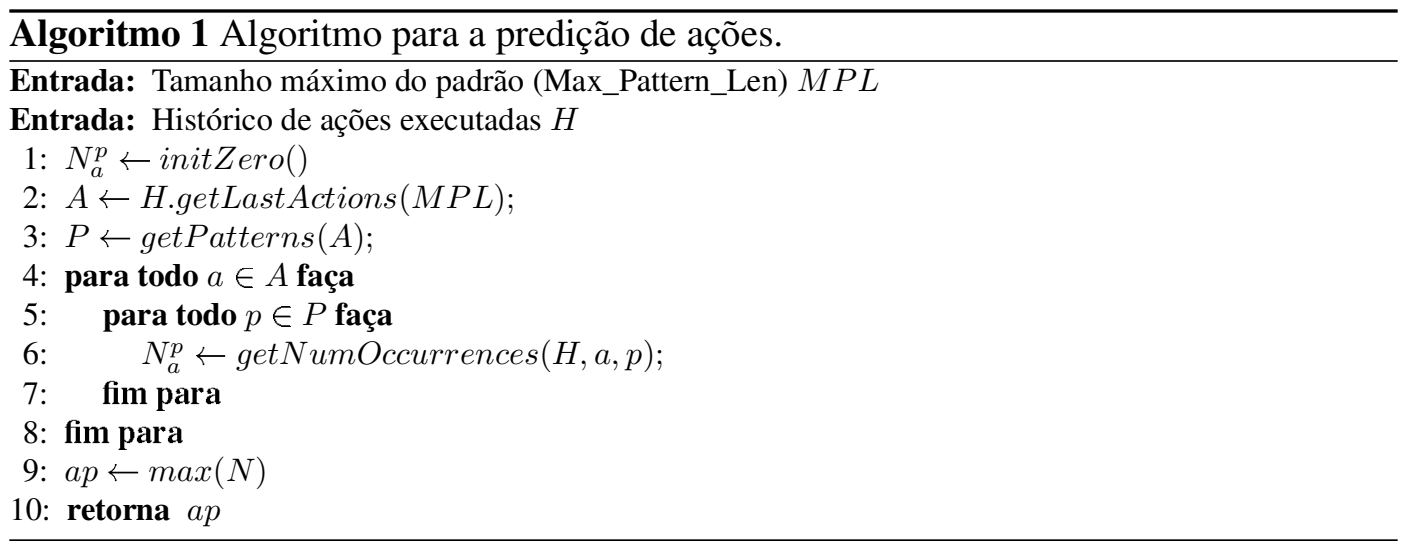

Assim, o algoritmo 1 busca a lista $A$ das últimas $M P L$ ações executadas, onde $M P L$ é o tamanho da janela ou a quantidade de ações a serem analisadas. Na sequência, é feita uma busca pela lista de padrões $P$ no histórico do usuário para cada uma das ações em $A$ anteriormente encontradas. Dessa forma, o número de ocorrências de cada uma das ações $a$, para cada padrão $p$ encontrado e relacionado em no máximo $M P L$ períodos com cada ação $a$ é atualizado. A maior ocorrência encontrada é a provável ação futura.

\subsection{Inferência do risco em ações}

A determinação de situações de risco em ações e atividades proposta nesse trabalho parte do pressuposto de que cada usuário possui seu próprio padrão de comportamento, na medida em que as pessoas são consideradas seres de hábitos [da Rocha et al. 2010]. Para 
determinar o risco, as mudanças que cada uma das atividades causam no contexto devem ser analisadas. Cabe ressaltar que essa análise deve ocorrer antes de uma ação ser executada pelo usuário. Ou seja, é necessário predizer as ações e atividades que podem ser executadas, e dessa forma identificar se o usuário estará em situação de risco caso execute determinada ação ou atividade. Essa abordagem implica na necessidade de $(i)$ capturar o contexto atual do usuário, (ii) inferir qual a próxima ação a ser executada, (iii) aplicar as mudanças de contexto decorrentes dessa ação no contexto atual e $(i v)$ avaliar o contexto resultante em busca de situações de risco.

Para alcançar esse objetivo, propôs-se um modelo de predição de ações, conjuntamente com um componente para descoberta das mudanças de contexto (Componente Mudança de Contexto) e um componente para simular contextos futuros (Visualizador de Contextos Futuros) decorrentes dessas mudanças. A partir da ação prevista, o Componente de Mudança de Contexto busca nos dados históricos do usuário quais as mudanças de contexto já causadas por ela. Por fim, o Visualizador de Contextos Futuros aplica as mudanças de contextos encontradas ao contexto atual do usuário com o objetivo de gerar um novo contexto que representa o estado futuro do contexto atual caso a ação predita for executada. Com base nesse contexto futuro, o Visualizador de Contextos Futuros pesquisa no perfil do usuário se ele está dentro dos limiares de segurança pré-estabelecidos para o usuário. Caso negativo, o usuário é considerado em uma situação de risco.

\subsection{Detecção de Atividade}

O modelo para a detecção de atividades parte do princípio que as atividades são compostas de ações, e que o relacionamento dessas ações determinam como uma atividade acontece. Dessa forma, se determinado conjunto de ações for executado em uma janela de tempo estabelecida, diz-se que uma atividade aconteceu. Para modelar as atividades cadastradas no sistemas, utilizou-se uma abordagem semelhante a de Naeem at al. (2007), a qual permite definir se ações são ou não obrigatórias, e se devem ser executadas em uma ordem definida.

Com isso, dois tipos de ações são definidos: $(i)$ ações núcleo e $(i i)$ ações secundárias. A primeira representa as ações que são essenciais para atingir o objetivo da atividade e, portanto, são necessárias para o seu reconhecimento. A segunda diz respeito àquelas relacionadas a uma determinada atividade, mas não essenciais para o seu reconhecimento, de modo que são úteis na medida em que acrescentam significado à atividade. Para que uma atividade seja detecta, cada ação que a compõe deve ser executada em uma janela de tempo pré-definida. Assim, uma janela de tempo inicial é determinada e na fase preliminar é ajustada para a calibração do sistema. Como esta janela depende da frequência de cada atividade, ela varia de acordo com o comportamento do usuário.

A Figura 4 apresenta um exemplo de reconhecimento de atividade. No exemplo, existem três atividades que o sistema é capaz de reconhecer: $X$, composta pelas ações $a_{1}$ e $a_{5}$, com uma janela de tempo de 3 períodos; $Y$, composta pelas ações $a_{2}, a_{3}$ e $a_{4}$, com uma janela de tempo de 5 períodos; e $Z$, composta pelas ações $a_{1}$ e $a_{4}$ com uma janela de tempo de 2 períodos. As ações detectadas em um certo período $t_{i}$ são dispostas dentro de uma caixa. Por exemplo, no período $t_{4}$ as ações detectadas são $a_{1}$ e $a_{7}$, pois a janela de tempo é considerada somente com as últimas ações detectadas. Dessa forma, é possível notar que a atividade $X$ foi detectada, uma vez que durante a sua janela de tempo as ações 
que a compõe foram executadas e detectadas $\left(a_{1}\right.$ em $t_{4}$ e $\left.a_{5} \mathrm{em} t_{5}\right)$. O mesmo acontece para a atividade $Y$, mas não para a atividade $Z$.

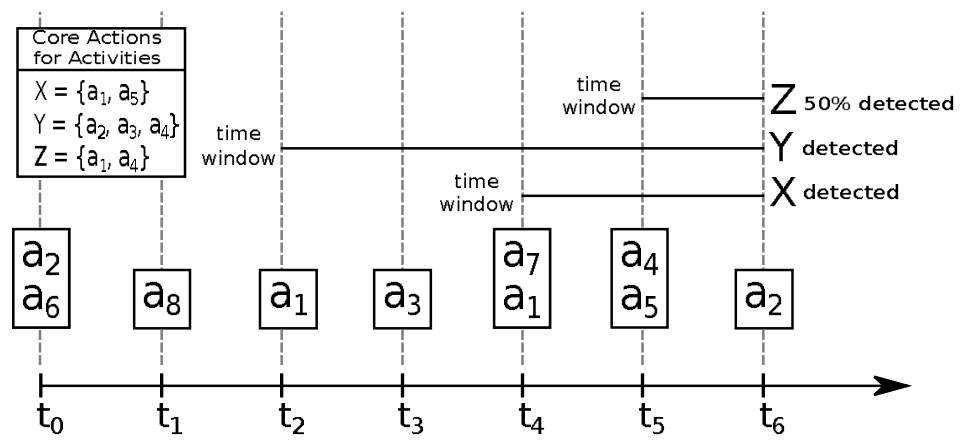

Figura 4. Exemplo do método para o reconhecimento de atividade baseado na TA.

Uma atividade é iniciada quando uma ação é detectada durante a janela de tempo, e considerada terminada com a detecção da última ação faltante que compõe a atividade. Se nem todas as ações forem detectadas durante a janela de tempo, a atividade é dita não completa, significando que somente algumas partes da atividade foram realizadas. Esse é o caso da atividade $Z$ na Figura 4. No exemplo apresentado, $X, Y$ e $Z$ estão acontecendo simultaneamente, embora a atividade $Z$ não esteja completa.

\section{Avaliação}

A detecção de situações de risco proposta neste trabalho baseia-se na predição das ações realizadas por um usuário baseado em seu comportamento prévio, bem como nas mudanças de contexto provocadas por esta ação. Neste sentido, é preciso que o sistema possa predizer as prováveis próximas ações executadas corretamente, bem como estimar se as mudanças de contexto ocasionadas por uma ação implicarão em um contexto arriscado para o usuário. Para validar a proposta deste trabalho, foram conduzidos dois experimentos distintos. O primeiro experimento visa analisar a precisão do modelo de predição apresentado, enquanto que o segundo visa validar o modelo para detecção de situações de risco baseadas nas mudanças de contexto provocadas pelas ações no contexto do usuário.

\subsection{Avaliação da predição de ações}

Para testar o modelo proposto neste trabalho, optou-se pela utilização de um dataset público, denominado Aruba Dataset [Cook 2011], pois permite que os resultados do modelo de predição de ações sejam comparados à outras propostas correlatas, visto que este é um dataset amplamente utilizado em pesquisas de reconhecimento de atividades na área de computação ubíqua. O dataset possui registrado 11 atividades diferentes utilizando 42 sensores. Assim, neste trabalho a precisão obtida a partir do dataset utilizado foi de $78,69 \%$, superior ao resultado obtido pelo algoritmo de grafos direcionais proposto por Gil-Quijano e Sabouret (2010), de 61,28\%, para o mesmo dataset. Isso representa um ganho de $28,41 \%$ no momento de inferir ações futuras.

\subsection{Avaliação da situação de risco em ações}

A avaliação de risco em ações foi realizada em um dataset próprio, pois não foram encontrados datasets públicos que contivessem informações relevantes, isto é, ações anotadas 
em conjunto com algum dado fisiológico do usuário. Neste sentido, o dataset é composto pelas ações caminhar, sentar, correr, deitar e ficar em pé, as quais foram detectadas a partir de dados de acelerômetro e giroscópio contidos em um smartphone. O dado fisiológico presente é a frequência cardíaca, coletada através de um sensor conectado a um Arduino.

Dessa maneira, das 2455 entradas, o modelo detectou 49 situações de risco e obteve uma precisão de $98,94 \%$. Esta precisão foi calculada com base nos valores verdadeiro-positivos (36), verdadeiro-negativos (2393), falso-positivos (13) e falsonegativos (13). Estes valores foram obtidos a partir da análise da comparação entre os valores para a frequência cardíaca atual, os limiares previstos para a ação atual e os limiares reais da ação atual. Os limiares previstos são determinados com base na ação diretamente anterior, de modo que é utilizada a mediana das mudanças de contexto realizadas por ela e o valor da frequência cardíaca enquanto ela era desenvolvida. Os limiares reais são medidos a partir da média dos valores históricos da frequência cardíaca para a ação atual e 3 vezes o desvio padrão destes mesmos valores, visto que, os limiares que consideram a média dos valores subtraída e adicionada 3 vezes do desvio padrão representa quase que a totalidade dos valores em uma distribuição normal.

\section{Conclusão}

A predição de situações de risco é importante para permitir que sistemas sensíveis ao contexto possam agir de forma preventiva, auxiliando na tomada de decisão do usuário. Assim, este trabalho apresentou um modelo para a predição de ações e detecção de situações de risco futuras, com base na Teoria da Atividade e no modelo Híperespaço Análogo ao Contexto. As técnicas utilizadas na abordagem adotada mostraram-se superiores a outros trabalhos relacionados, visto que, para a predição de ações, obteve-se uma taxa de precisão de 78,69\% e para a avaliação de situações de risco, obteve-se uma precisão de $98,94 \%$. Como trabalhos futuros pretende-se melhorar a abordagem para predição de ações, considerando a avaliação da performance dos algoritmos. Além disso, pretende-se realizar testes em datasets com um maior número de informações fisiológicas do usuário, para permitir uma avaliação mais completa dos riscos calculados.

\section{Referências}

Cook, D. (2011). Learning setting-generalized activity models for smart spaces. IEEE Intelligent Systems.

Crispim-Junior, C. F., Bremond, F., and Joumier, V. (2012). A multi-sensor approach for activity recognition in older patients. In The Second International Conference on Ambient Computing, Applications, Services and Technologies-AMBIENT.

Curone, D., Tognetti, A., Secco, E. L., Anania, G., Carbonaro, N., De Rossi, D., and Magenes, G. (2010). Heart rate and accelerometer data fusion for activity assessment of rescuers during emergency interventions. Information Technology in Biomedicine, IEEE Transactions on, 14(3):702-710.

da Rocha, C. C., Lima, J. C. D., Viera, M., Capretz, M. A., Bauer, M. A., Augustin, I., and Dantas, M. A. (2010). A context-aware authentication approach based on behavioral definitions. In IKE, pages 178-184. 
Del Fabro Neto, A., Boufleuer, R., Romero de Azevedo, B., Augustin, I., and Carlos D. Lima, J. (2014). Modelo para classificação do esforço cognitivo de atividades humanas em um sistema sensível ao contexto. Proceedings of VI Simpósio Brasileiro de Computação Ubíqua e Pervasiva, SBCUP.

Del Fabro Neto, A., Boufleuer, R., Romero de Azevedo, B., Augustin, I., Carlos D. Lima, J., and C. Rocha, C. (2013). Towards a middleware to infer the risk level of an activity in context-aware environments using the srk model. In UBICOMM 2013, The Seventh International Conference on Mobile Ubiquitous Computing, Systems, Services and Technologies, pages 38-42.

Dernbach, S., Das, B., Krishnan, N. C., Thomas, B. L., and Cook, D. J. (2012). Simple and complex activity recognition through smart phones. In Intelligent Environments (IE), 2012 8th International Conference on, pages 214-221. IEEE.

Gil-Quijano, J. and Sabouret, N. (2010). Prediction of humans' activity for learning the behaviors of electrical appliances in an intelligent ambient environment. In Web Intelligence and Intelligent Agent Technology (WI-IAT), 2010 IEEE/WIC/ACM International Conference on, volume 2, pages 283-286. IEEE.

Kuutti, K. (1996). Activity theory as a potential framework for human-computer interaction research. pages 17-44.

Lu, C.-H. and Fu, L.-C. (2009). Robust location-aware activity recognition using wireless sensor network in an attentive home. volume 6, pages 598-609. IEEE.

Mikalsen, M. and Kofod-Petersen, A. (2004). Representing and reasoning about context in a mobile environment. pages $25-35$.

Mocanu, I. and Florea, A. M. (2011). A model for activity recognition and emergency detection in smart environments. In AMBIENT 2011, The First International Conference on Ambient Computing, Applications, Services and Technologies, pages 13-19.

Naeem, U., Bigham, J., and Wang, J. (2007). Recognising activities of daily life using hierarchical plans. In Smart Sensing and Context, pages 175-189. Springer.

Rasch, K. (2013). Smart assistants for smart homes. PhD thesis, Royal Institute of Technology.

Silva, T. H., Celes, C., Mota, V., and Loureiro, A. (2012). Overview of ubicomp research based on scientific publications. Proceedings of IV Simpósio Brasileiro de Computação Ubiqua e Pervasiva, SBCUP.

Vygotsky, L. S. (1980). Mind in society: The development of higher psychological processes. Harvard university press.

Wang, C., Zheng, Q., Peng, Y., De, D., and Song, W.-Z. (2014). Distributed abnormal activity detection in smart environments. International Journal of Distributed Sensor Networks, 2014. 